\title{
UN MÉTODO PARA DETERMINAR COMPETENCIAS DISTINTIVAS EN PEQUEÑAS Y MEDIANAS EMPRESAS
}

\author{
A METHOD TO DETERMINE CORE COMPETENCES \\ IN SMALL AND MEDIUM ENTERPRISES
}

Recebido - 29/03/2011

Aceito - 10/07/2011

Carlos M. F-Jardon ${ }^{1}$ e María Susana Martos ${ }^{2}$

\section{Resumen}

Las fuentes de ventajas competitivas son características de las empresas que les permiten situarse en mejor posición que sus competidores. Las modernas teorías de la empresa consideran que esas fuentes se encuentran en sus factores internos y externos. La gerencia, mediante la estrategia combina dichos factores estableciendo sus competencias distintivas. Este trabajo propone un método para determinar la formación de dichas competencias. Se discuten las diferentes técnicas utilizadas y se propone una alternativa basada en componentes principales con rotación oblimin directa. Se evalúa la aplicación de este método a un caso particular en Pymes.

Palabras clave: Fuentes de ventaja competitiva. Competencias distintivas. Pymes. Componentes principales.

\footnotetext{
${ }^{1}$ Departamento de Economía Aplicada. Universidad de Vigo. E-mail: cjardon@uvigo.es

2 Departamento de Organización de Empresas y Marketing. Universidad de Vigo. E-mail: smartos@uvigo.es
} 


\section{Summary}

The sources of competitive advantage are firm characteristics that allow them to be in a better position than its competitors. Modern firm theories consider that these sources are internal and external factors of business. Entrepreneur combines these factors by the strategy to set their core competencies. This paper proposes a method for determining the formation of core competencies. We discuss the different techniques used and we propose an alternative based on principal components with direct oblimin rotation. We assess the implementation of this method to a particular case in SMEs.

Keywords: Sources of competitive advantage. Core competencies. Regional SMEs. Principal components.

\section{INTRODUCCIÓN}

En América latina, la competitividad ha sido estudiada tanto en el campo nacional (KETELHÖHN; MONCAYO; ALLEN, 1999) como en el campo empresarial (ARTEAGA; LASIO, 2009). En este último la competitividad está asociada a la idea de competencias distintivas que son las que generan ventajas competitivas.

En principio, cualquier característica de la empresa podría ser fuente de ventaja competitiva (FVC). En general se han considerado que las FVC se encuentran tanto en los factores internos como externos de la empresa (GRANT, 2005). Partiremos de un modelo que enlaza los factores internos y externos de la empresa con sus competencias distintivas. En la mayoría de los trabajos las competencias son estudiadas como constructos de un segundo o tercer nivel dependiendo de los autores (BARNEY, 1996; GRANT, 1991), es decir, que no son variables directamente observables, sino que son construidas combinando una serie de características de la empresa. Por ese motivo en la mayor parte de los trabajos, las competencias son analizadas como constructos multivariantes, que no se pueden medir de modo directo por una única variable (CARSON; GILMORE, 2000; RUBIO; ARAGÓN, 2008). Por tanto es habitual hacer uso de técnicas multivariantes para determinar las competencias distintivas. (RUBIO; ARAGÓN, 2008; PÉREZ; PRIETO; MARTíN, 2009; HUGHES; MORGAN, 2008; MALAVER; VARGAS, 2004).

Existen numerosos trabajos que detallan dichas competencias, aunque no se presenta una metodología común a todos ellos y eso hace difícil la comparación entre unos y otros (BONTIS; KNOW; RICHARDSON, 2000; RAY; BARNEY; MUHANNA, 2004; JARDON; MARTOS, 2010). Además, las pymes presentan particularidades que merecen especial atención (VAONA; PIANTA, 2008). Una simplificación habitual es hacer uso de métodos multivariantes lineales (RUBIO y ARAGÓN, 2008; DÍAZ; PÉREZ, 2002).

Dentro de esas técnicas se presentan dos aproximaciones, que dependen en gran parte del enfoque que se realiza para llevar a cabo el estudio. La primera es conocida como análisis confirmatorio y propone a priori los constructos que caracterizan las competencias, definiendo las características que mejor integran ese constructo (BONTIS; KNOW; RICHARDSON, 2000; GATTERMANN et al., 2010; HUGHES; MORGAN, 2008). La segunda se denomina análisis exploratorio (COSTELLO; OSBORNE, 2005; JARDON; MARTOS, 2010) y busca la relación entre los ítems previamente definidos y los constructos obtenidos finalmente.

Un problema adicional que se plantea está asociado a la interpretación de los factores obtenidos. Normalmente se hace uso de una serie de técnicas de rotación que permiten ajustar los ejes, permitiendo de esa forma interpretar mejor el significado de los constructos obtenidos. 
Sin embargo, dependiendo de la rotación elegida es posible que cambie la interpretación de los factores y las características asociadas a cada competencia. La mayoría de los paquetes informáticos proponen por defecto métodos de rotación ortogonales que no se ajustan a lo esperado, ya que las competencias suelen estar relacionadas entre sí.

En este trabajo, intentaremos dar respuesta a estos interrogantes, justificando la elección de un método para determinar las competencias distintivas de una empresa. De esa forma, propondremos una metodología que permitirá analizar como la gerencia organiza las diferentes FVC para establecer sus competencias distintivas, facilitando la comparación entre trabajos.

Este trabajo busca una particular aplicación a pymes latinoamericanas. Los métodos factoriales han sido utilizados en gran número de problemas de Economía y Empresa (DÍAZ; PÉREZ, 2002; CONFORTINI; LOPEZ, 2009) y además existen buenos manuales que explican su uso y dan una justificación teórica de su necesidad (HARMAN, 1976; GORSUCH, 1983; HAIR et al., 2006). Sin embargo, es menos habitual un trabajo que justifique el uso de dichos métodos aplicados a la determinación de competencias distintivas, si bien en la práctica ha sido muy utilizado (CARSON; GILMORE, 2000; RUBIO; ARAGÓN, 2008). Al igual que cada problema del mundo económico necesita una determinada técnica multivariante, la determinación de competencias distintivas exige una técnica específica dentro de los diferentes métodos factoriales. Eso no quiere decir que sea la única, pues depende de las suposiciones del modelo utilizado. De acuerdo a esas suposiciones expondremos cuales pueden ser las mejores prácticas en las diferentes alternativas.

Dividiremos este artículo en tres apartados. En el primero, se discuten los principios metodológicos que justifican la metodología propuesta. A continuación se desarrolla un caso de aplicación de esa metodología. Para ello se analizan las competencias distintivas de Pymes de una zona del noroeste de España y se discuten los resultados. Se termina con un apartado de conclusiones.

\section{DETERMINACIÓN DE COMPETENCIAS DISTINTIVAS}

Según Prahalad y Hamel (1991), las competencias distintivas son aquellas que hacen una contribución desproporcionada al valor para el cliente final, o a la eficiencia con que dicho valor se obtiene y proporcionan una base para entrar en nuevos mercados. Es decir, generan ventajas competitivas en la empresa. En general, una ventaja competitiva se define como aquel aspecto de la empresa no imitable fácilmente, con posibilidades de ser mantenido en el futuro, en el que se posiciona por encima de sus competidores y que le lleva a obtener mejores resultados. (CARMELI, 2004). Existen tres elementos asociados a este concepto: una combinación de características de la empresa que se mantiene en el tiempo y difícilmente imitable, una comparación con los competidores y una utilidad práctica que se manifiesta en obtener, de alguna manera, mejores resultados empresariales. (O'DONNELL et al., 2002).

El primer paso para construir una ventaja competitiva consiste en definir las posibles fuentes que le permitan situarse en una mejor posición respecto a sus competidores. En principio, cualquier característica de la empresa podría ser FVC. Han sido numerosos los modelos de estudio de estos enfoques. En general comparten que las fuentes de ventajas competitivas se encuentran tanto en los factores internos como externos de la empresa. (GRANT, 2005; GRANT, 1991; BARNEY, 1996). Partiremos, por tanto, de un modelo que enlaza los factores internos y externos de la empresa con sus ventajas competitivas. 
Aunque las FVC para las empresas son comunes, no ocurre lo mismo en la forma en que éstas las organizan. Esto hace que en la práctica sean diferentes los modelos de contraste de ventajas competitivas, puesto que no suelen ir asociadas directamente a un único recurso o capacidad sino que suelen tener en cuenta una combinación de ellos (GILMORE; CARSON, 1999). Dicha combinación se hace mediante la estrategia. (GRANT, 2005). Eso significa que las competencias son estudiadas como constructos de un segundo o tercer nivel, que obliga a hacer uso de técnicas multivariantes para determinarlas.

En la práctica, se suele reducir el conjunto de métodos posibles a los métodos lineales. El argumento utilizado para ello es clásico en las ciencias sociales. Dado que las competencias distintivas son funciones de una serie de características de la empresa, (BARNEY, 1996; GRANT, 2005) se puede suponer que dicha función es continua. Dichas funciones se pueden aproximar por funciones lineales. Esto nos justifica el hecho de centrarnos únicamente en los métodos lineales.

También se asume que el grado de incertidumbre de los datos viene dado por la variabilidad total de estos, puesto que cuanto mayor sea ésta, más difícil es predecir o estimar un valor concreto. Al conocer información sobre el modelo, su variabilidad se reduce y en consecuencia, se está reduciendo el grado de incertidumbre. Por consiguiente se puede suponer que la información de cada una de esas FVC viene medida por su grado de variabilidad.

Una vez asumidos esos principios, se debe elegir entre las diferentes técnicas lineales multivariantes. Dentro de estas existen dos enfoques diferentes según se presupongan conocidas las hipótesis del modelo o se busque descubrir cuáles son éstas. El primer enfoque es conocido como análisis confirmatorio. Este propone a priori las competencias, definiendo las características que mejor las integran. (HUGHES; MORGAN, 2008; GATTERMANN et al., 2010). Ese enfoque, normalmente, no busca determinar cuáles son las FVC, sino evaluar directamente los contenidos de las competencias. No obstante, en algunos casos presuponen que existe una relación causal entre las variables previamente definidas y las competencias y, en ese sentido, las primeras podrían considerarse como FVC. El proceso econométrico de inferencia confirma que esas competencias están bien definidas (HAIR et al., 2006), pero no explicita si esas variables establecidas por los ítems son realmente FVC. Lo más habitual suele ser indicar que existe esa relación y que debe ser la gerencia la que determine como seleccionar sus recursos, capacidades y factores externos para asociarlos entre sí constituyendo una nueva competencia distintiva. (HAFEEZ; ZHANG; MALAK, 2002; GRANT, 2005).

En cualquiera de los casos anteriores, existe un problema asociado a los métodos confirmatorios. Las variables que se establecen para cada competencia no suelen compartirse con otra competencia. Esto significa que el empresario asigna de modo único cada potencial FVC a una única competencia, cuando hay características, como por ejemplo el capital humano, que normalmente se utilizan para diferentes competencias distintivas. En consecuencia, esa metodología no parece muy realista al condicionar en la práctica las decisiones empresariales. Por este motivo este tipo de análisis son utilizados fundamentalmente como forma de evaluar el impacto de competencias distintivas sobre algún aspecto o viceversa, pero no como métodos de determinación de competencias distintivas. Por todo ello parece lógico buscar dentro del análisis exploratorio para localizar una metodología que permita determinar la formación de competencias distintivas. Esta aproximación busca la relación entre los ítems previamente definidos y los constructos obtenidos finalmente. En ese sentido se puede hablar de relación entre ambos si las variables iniciales miden características que son posibles FVC y el constructo final obtenido se puede considerar una competencia distintiva. Esta justificación es asumida también para otros aspectos de las ciencias sociales. (COSTELLO; OSBORNE, 2005). 
En muchos trabajos, se hacen uso de técnicas multivariantes de segunda generación como LISREL (HAIR et al., 2006) o PLS (CHIN, 1998), pero estas técnicas están orientadas a la resolución de un problema diferente. Estas técnicas buscan demostrar que existe causalidad entre dos o más variables latentes a partir de la información suministrada por una serie de variables manifiestas. Consecuentemente, no trataremos esas técnicas en este trabajo. Nos centraremos únicamente en las técnicas lineales de reducción de información para determinar las competencias.

Dentro de estos métodos, es común comparar dos modos alternativos, si bien comparten algunos principios similares. El primero es conocido como análisis factorial; el segundo es el análisis de componentes principales (ACP). Ambos se engloban dentro de los denominados métodos factoriales. (LAWLEY; MAXWELL, 1962). En ambos métodos existen elementos comunes. Uno de ellos es que la solución matemática obtenida no es única. Este hecho es el que permite realizar rotaciones para interpretar mejor los resultados obtenidos.

El planteamiento clásico del análisis factorial se basa en presuponer que las variables latentes son la fuente principal de variación de las variables manifiestas. En esencia presupone que las competencias se definen en un espacio común a las $\mathrm{FVC}$, que recoge la información que tienen en común dichas FVC, dejando un error, que es consecuencia de la información más específica de cada variable que no es común con el resto.

Por tanto este planteamiento indica que las FVC observadas son causadas por las competencias no observables directamente, es decir, lo inverso de lo que buscamos. Sin embargo, es fácil por transformación de datos, justificar la relación inversa. Esto hace que los modelos de análisis factorial sean asequibles para ser utilizados como forma de estimar las competencias de la empresa. La dificultad añadida a este método es consecuencia de las suposiciones en las que se basa para obtener estimadores más eficientes. Para ello hace uso del método de máxima verosimilitud, método que exige la normalidad de las variables manifiestas de modo conjunto. En la práctica esta suposición es difícil de verificar y más, cuando se trabaja con escalas de Likert, como suele ser habitual en los trabajos sobre competencias. Esto dificulta el uso práctico de este método.

Otra alternativa es el uso de componentes principales. Esta técnica consiste en reducir la dimensión del conjunto de ítems inicial buscando la información común a todos ellos y creando unas nuevas variables que recogen esa parte de información común quedando de modo residual la información que podría considerarse más específica de cada una de los ítems originales.

Si bien este es un método de reducción de información, hay varios argumentos que justifican que las competencias distintivas se obtengan mediante componentes principales. La idea fundamental se basa en el propio concepto de competencia distintiva. Parece lógico buscar una combinación de FVC que maximice el uso de su información para generar el mayor valor posible al cliente. Es decir, la competencia debería hacer uso de la mayor cantidad de información disponible en la empresa, para aprovecharla de la manera más eficiente posible. (BERMAN; DOWN; HILL, 2002). Por consiguiente el problema se reduce a determinar cuál es la relación entre las FVC y las competencias distintivas, que minimiza la información perdida. Este es un problema clásico de métodos factoriales, donde el espacio reducido viene dado por las componentes principales. (HARMAN, 1976).

El ACP busca sólo reducir el espacio perdiendo la mínima información posible. El análisis factorial busca encontrar cual es el espacio común a las variables manifiestas. La diferencia esencial se encuentra en el método de obtención de los factores. Mientras que en el primero se supone que la matriz de correlaciones es completa, en el segundo se suaviza ésta, sustituyendo la diagonal principal por las comunalidades. Cuando las comunalidades son altas (mayores que 
0.6) todos los procedimientos tienden a dar la misma solución. Además, si el número de variables es alto (mayor que 30), las estimaciones de la comunalidad tienen menos influencia en la solución obtenida y en consecuencia, todos los métodos tienden a dar el mismo resultado. (HARMAN, 1976).

Por tanto, cualquier método es válido desde el punto de vista práctico para estimar los resultados. (STEWART, 1981). El único problema puede ser la falta de convergencia del método utilizado. Desde el punto de vista teórico depende de las suposiciones subyacentes. Es más robusto, utilizar un método de componentes principales, básicamente descriptivo, que no exige ninguna suposición inferencial, pero los resultados son menos generalizables, ya que dependen de los datos utilizados. Además, hay que tener en cuenta que todos estos resultados están condicionados por el tamaño muestral.

\subsection{El número de competencias}

Un segundo problema surge cuando se trata de determinar cuántas competencias se generan en la empresa, es decir, cual es la dimensión del espacio de competencias. La mayor parte de los paquetes consideran por defecto el método de normalización de Guttman-Kaiser que presupone que el número de factores elegido debe ser igual al número de autovalores mayores que 1 existentes en los datos. Intuitivamente el argumento se basa en que no haya ninguna componente «explicando» menos que la varianza de una variable original. (GUTTMAN, 1954). Utilizarlo como único criterio ha sido criticado en la literatura puesto que suele sobreestimar el número de factores del modelo. (VELICER; JACKSON, 1990).

Diferentes autores sugieren hacer uso del gráfico de sedimentación. (COSTELLO; OSBORNE, 2005). Este gráfico representa el valor de cada autovalor respecto al orden en el que se introduce esa componente en el modelo. Cada autovalor mide la variabilidad de cada factor. Por consiguiente, ese gráfico nos indica la parte de información que aporta cada componente al modelo. De ese modo, altos valores del autovalor indican mucha información común recogida por él. Por tanto, mientras se introduzcan componentes que recojan esa estructura común, los autovalores serán altos. En el momento que se empiecen a introducir componentes con poca información los autovalores decaerán mucho respecto a los anteriores y tenderán a estabilizarse, puesto que aportan información similar todos ellos. Consecuentemente, cuando se presenta una estructura factorial dentro de los datos se producirá un cambio sustancial en el gráfico. Se suele utilizar como número ideal de factores el valor en el cual se produce el último cambio abrupto, que nos indica que a partir de ese valor se "sedimenta" la aportación de los nuevos factores al modelo.

Otro criterio utilizado con cierta frecuencia desde el campo de las aplicaciones es la cantidad de varianza acumulada explicada por los factores, puesto que nos está diciendo la parte de información que tiene el modelo resumido respecto a la información total. No interesa que se pierda mucha información puesto que entonces seria síntoma de que no se están utilizando de modo eficiente las FVC. En ese sentido, dependiendo de las diferentes disciplinas sociales cambian las exigencias de variación explicada por el modelo, desde un $60 \%$ en las ciencias sociales (HAIR et al., 2006) hasta más de un 40\% en Sicología. (TINSLEY; TINSLEY, 1987). En nuestro caso, al referirse a competencias distintivas parece conveniente exigir un mínimo del 50\%. 
Dada toda esa variedad de posibilidades, parece conveniente hacer uso de todos los criterios junto con la interpretación final de los factores a la hora de decidir cuál es el mejor número de competencias. (HARMAN, 1976; STEWART, 1981). El proceso que se sugiere es el siguiente: Inicialmente, se hace uso del grafico de sedimentación. Este nos indicará el mínimo número de factores que debemos introducir en el modelo. A continuación, se va extendiendo el número de los factores hasta que se cubra al menos un $50 \%$ de la variación explicada y como máximo hasta incluir todos los factores que tienen autovalores mayores que 1. Una vez llegados a este punto se debería determinar si las variables verifican los criterios de inclusión en el modelo que se analizarán posteriormente. En caso contrario se eliminarían esas variables y se reiniciaría el proceso. En cualquier caso, después de la interpretación final de los factores se revisaría ese número por si interesa un ajuste posterior.

\subsection{Medidas de evaluación}

El grado de validez de la aplicación de los métodos factoriales viene dado por dos instrumentos auxiliares: el test de Bartlett y el coeficiente de Kaiser- Meyer y Okin (KMO). El primero contrasta si la matriz de correlaciones entre los ítems originales es una matriz identidad, es decir, no existe información común entre dichos ítems y, por lo tanto, no se puede buscar esa información. Por tanto, dicho test debe ser significativo lo que indicaría que existe información común entre los ítems analizados. (ANDERSON, 1963). Sin embargo, este test exige la normalidad de las variables manifiestas que es poco frecuente en el caso de escalas de Likert. No obstante, existen alternativas de test asintóticos para componentes principales. (BAI, 2003).

El segundo instrumento mide la adecuación muestral comparando las correlaciones parciales entre los ítems que intervienen. Si dicho coeficiente es cercano a 1 es un indicador de que las correlaciones parciales son prácticamente nulas y por consiguiente, la información de los ítems se encuentra comprendida en el conjunto de todos ellos, es decir las especificidades de cada ítem son pequeñas en relación al conjunto. Los valores que se suelen considerar como aceptables son aquellos mayores de 0,6. (KAISER, 1974). Ambos fueron instrumentos ideados para el análisis factorial, pero mantienen una cierta validez para el ACP, cuando estas se utilizan como técnicas de reducción de la información.

Para evaluar si la matriz de correlaciones se adapta a los factores se hace uso de la matriz anti-imagen. Esta señala la parte que es única en cada variable, es decir, la parte que no se puede predecir a partir de las demás variables. Cuando los elementos de fuera de la diagonal son cercanos a cero indica que el modelo se ajusta bien. (KAISER, 1963).

Otra técnica utilizada en algunos trabajos es el coeficiente Alfa de Cronbach. (CRONBACH, 1951). Este coeficiente está más orientado a indicar si unos ítems predeterminados e incluidos en un cuestionario con idea de medir una misma variable latente, realmente son consistentes entre sí para este propósito y no para evaluar si unas variables manifiestas afectan a una latente. Por ese motivo no parece necesario hacer uso de él para buscar competencias distintivas.

Para evaluar si una característica es FVC se hace uso de la comunalidad. Esta indica la parte de la varianza de la variable explicada por el modelo, es decir, la parte de la FVC que es utilizada para elaborar las competencias distintivas.

Cuando una característica tiene una comunalidad muy baja quiere decir que apenas se utiliza para elaborar competencias distintivas y por consiguiente puede ser eliminada de las 
FVC. Se consideran comunalidades altas si son mayores de 0,8 (VELICER; FAVA, 1998), pero esto difícilmente se consigue en todas las variables con datos reales. Por ello se suele asumir que variables con comunalidades mayores de 0,4 pueden ser asumidas. (COSTELLO; OSBORNE, 2005). Este será uno de los criterios para indicar cuando una variable se puede considerar FVC.

Las competencias distintivas no se asocian de modo directo a las componentes principales obtenidas, sino que es necesario rotar los ejes para interpretar mejor el sentido de esas componentes. Es aquí donde se presentan las mayores discrepancias metodológicas.

\subsection{Rotaciones de componentes principales}

La justificación de esta técnica se basa en presuponer que si se mantiene la dimensión del espacio, no se pierde información y, por lo tanto, el nuevo espacio trasformado es equivalente al anterior.

Se intenta obtener una estructura factorial lo más simple posible, es decir, que la interpretación de cada factor sea lo más unívoca posible. Esto se facilita si cada variable se asigna a un único factor. Además se debe exigir que todos los factores tengan dos o más variables asignadas (THURSTONE, 1947), puesto que sí aparece una única característica en un factor, será un indicador de que esa característica no está relacionada con el conjunto y, por lo tanto no se combina para formar competencias distintivas.

La idea de asignación única presenta dos conceptos en los que no existe un acuerdo definitivo. El primero se refiere a la carga cero que se puede considerar que no afecta al factor. Se suelen definir como aquellas cuyos valores se sitúan entre -0,10 y +0,10. (GORSUCH, 1983). El segundo se refiere al concepto opuesto denominado carga significativa. Su valor depende del tamaño muestral. En la práctica, los investigadores suelen tomar las cargas mayores de 0.30 (KLINE, 2002), o de 0,32 (TABACHNICK; FIDELL, 2001) puesto que esta cantidad señala que dicha variable aporta aproximadamente un $10 \%$ de la varianza del factor. Dentro de esta misma idea, a la hora de interpretar el sentido de los factores se introduce el concepto de carga fuerte (COSTELLO; OSBORNE, 2005). Esta se define cuando su valor es mayor o igual a 0,50.

Cuando una variable aparece en más de un factor se dice que es una variable compleja. Este no sería un problema a la hora de hablar de competencias, puesto que es fácil que una característica intervenga en más de una competencia.

Lo deseable para que un factor se pueda considerar sólido es que tenga al menos 5 cargas factoriales fuertes, puesto que cuando tiene menos de 3 cargas factoriales fuertes generalmente es débil e inestable y, en consecuencia, sus resultados son menos fiables. Eso exige que el número de variables sea grande en relación al número de factores.

Normalmente, no es posible conseguir estructuras de ese tipo de forma directa mediante rotación, puesto que partiendo de una matriz de relación entre factores y variables, o bien se optimiza el tamaño de las cargas en las variables (filas), sin saber que ocurre con los factores (columnas), o viceversa. Por ese motivo existe una diversidad de métodos de rotación.

Por construcción, los ejes obtenidos por ACP son ortogonales. Al hacer la rotación se puede exigir que esos ejes rotados sigan siendo ortogonales, pero no es necesario. Estas da lugar a dos formas básicas de realizar la rotación de factores: la Rotación Ortogonal y la Rotación Oblicua. Cuando los factores son ortogonales, las cargas factoriales coinciden con las correlaciones entre las variables observadas y los factores y, en consecuencia, se pueden utilizar estas de modo directo para interpretar los factores. Cuando la rotación es oblicua esto no ocurre, generando 
un problema adicional de selección de la matriz mejor para interpretar los factores. Por este motivo, en la práctica ha sido común asumir rotaciones ortogonales para evitar este problema.

Tanto en la rotación ortogonal como en la oblicua la comunalidad de cada variable no se modifica. Sin embargo, cambia la varianza explicada por cada factor, luego los nuevos factores rotados no están ordenados de acuerdo con la información que contienen, cuantificada a través de su varianza.

El método utilizado por defecto en la mayoría de los paquetes informáticos es la Rotación varimax. Este es un método de rotación ortogonal que minimiza el número de variables que tienen saturaciones altas en cada factor. Simplifica la interpretación de los factores y tiende a producir grupos de factores múltiples. En cada factor las cargas grandes se incrementan y las pequeñas se disminuyen, de manera que cada factor solo tiene unas pocas variables con cargas grandes. Sin embargo, el mantenimiento de ortogonalidad a menudo dificulta su interpretación.

Otro método de rotación ortogonal que, al contrario que el varimax, maximiza la varianza de las cargas factoriales al cuadrado en cada variable, es decir, simplifica las filas de la matriz de cargas factoriales, es la Rotación quartimax. Este tipo de rotación sesga la introducción de las cargas hacia los primeros factores, en los que tienden a aparecer más cargas fuertes que en los demás. En consecuencia, tiende a producir un factor general y un pequeño grupo adicional de factores múltiples. Dificulta la interpretación de los factores pero facilita la asignación de cada característica a una determinada componente, justificando los estudios realizados mediante técnicas confirmatorias. Esto es una consecuencia de la forma de optimizar las cargas que utiliza este método y, no parece ser conveniente su uso, salvo que nos interesen pocas competencias y muy complejas.

Este método es utilizado con frecuencia como comprobante puesto que si las rotaciones varimax y quartimax son muy diferentes posiblemente exista una estructura factorial muy débil.

Finalmente, combinando los dos métodos anteriores se obtiene el Método equamax. Este método maximiza una suma ponderada de los criterios varimax y quartimax, lo que refleja una preocupación por la estructura simple dentro de las variables, así como en factores. Su interpretación suele ser muy similar al método varimax y sólo se utiliza cuando este no da una interpretación clara.

Todos estos métodos de rotación presentan un problema esencial a la hora de definir competencias distintivas. Al exigir la ortogonalidad, estamos imponiendo que las competencias no tienen nada que ver unas con otras, y esto en la práctica parece difícilmente sostenible. Por eso tiene más interés hacer uso de métodos de rotación oblicuos. Stewart (1981) sugiere algo similar para estudiar el comportamiento de los consumidores.

El más común entre estos últimos es el método Oblimin Directo, que en realidad no es un único método sino un conjunto de alternativas posibles pues hace uso de un parámetro (Delta) que al incrementarse, aumenta el grado de oblicuidad. A la hora de seleccionar el valor de Delta, se pueden considerar los criterios clásicos de Thurstone para decidir entre diferentes rotaciones. (BROWN, 2009). Inicialmente parece lógico empezar con un Delta igual a 0, puesto que es un valor intermedio y luego ir aumentándolo de acuerdo a las posibles interpretaciones.

Este método tiende a producir las mismas soluciones que la rotación varimax pero permite que sean oblicuas, lo que facilita su interpretación y uso práctico. Sin embargo, presenta una dificultad. Al hacer una rotación oblicua se obtienen dos matrices diferentes que se pueden utilizar para la interpretación: la matriz de estructura y la matriz de configuración. La matriz de estructura contiene las correlaciones entre cada variable y cada factor (igual que con las rotaciones ortogonales). Por su parte, la matriz de configuración contiene los cargas factoriales para reproducir las variables de puntuaciones de los factores. 
Existe un considerable desacuerdo sobre cuál de ellas es la mejor para la interpretación de los factores. El uso de la matriz estructura se basa en la larga historia de interpretación de factores en términos de las variables con las que se correlacionan. (PETT; SULLIVAN; LACKEY, 2003; HARMAN, 1976). El uso de la matriz de configuración se basa en que suele tener una estructura más simple. (HAIR et al., 2006). No obstante esa simplicidad puede ser aparente ya que se consigue gracias a la corrección de colinealidad por las cargas factoriales. (PETT; SULLIVAN; LACKEY, 2003). Con suerte, la interpretación basada en las dos matrices será similar. En la práctica suele ser conveniente tener en cuenta los dos elementos para realizar la interpretación puesto que ambos aportan información sobre el comportamiento del factor.

Finalmente, cuando se tiene un conjunto grande de datos se suele utilizar la Rotación Promax, que es una rotación oblicua que tiende a producir factores similares a los de varimax y oblimin directo, pero que se puede calcular más rápidamente.

Suele ser difícil decidir entre una metodología u otra. Siempre hay que tener en cuenta las condiciones teóricas del modelo que se analiza. En el caso de las competencias, parece lógico hacer uso de rotaciones oblicuas. A la hora de elegir la matriz para la interpretación, nuestra sugerencia es hacer uso de la matriz de configuración para una interpretación previa de los factores, puesto que tiende a establecer estructuras más simples. Sin embargo a la hora de evaluar qué características afectan a una determinada competencia se debe considerar la matriz de estructura, puesto que esa relación viene medida por las correlaciones. Este efecto complementario puede cambiar en algún aspecto la interpretación de los factores realizada previamente.

\section{METODOLOGÍA PARA EL PROCESO DE COMPARACIÓN}

El objetivo de este trabajo consiste en comparar los diferentes métodos analizados para determinar la formación de competencias distintivas para la empresa y evaluar sus diferencias, de manera, que se puede establecer unas buenas prácticas en dicho proceso. Una posible alternativa sería hacer una simulación y comprobar cómo se comporta cada una de las metodologías en dicha simulación. Sin embargo, en una simulación, el modelo es diseñado a priori y, por consiguiente, el método que mejor se adapte será aquel que cumpla los requisitos del modelo diseñado. Por ello, parece más interesante hacer uso de un caso real y establecer algunas buenas prácticas alrededor de él. Interesa determinar cómo se combinan una serie de recursos y capacidades con los factores externos para construir sus potenciales ventajas competitivas.

En primer lugar, comprobaremos la forma de la distribución de los datos para optar por el análisis factorial o por el ACP. En caso de validez de ambos se hará una comparación de sus resultados. Para el proceso de determinación de factores y evaluación de efectos se utilizó el programa Statistical Package for the Social Sciences (SPSS versión 15).

Uno de los objetivos del trabajo era proponer un método práctico válido para pymes latinoamericanas. El funcionamiento de estas pymes es muy similar en sus aspectos culturales al de las pymes gallegas. (JARDON et al., 2007). Por ello se ha buscado un trabajo sobre Competencias distintivas en Vigo y su área metropolitana en el año 2005 (JARDON; MARTOS, 2010) para obtener los datos. Esta zona se encuentra en el noroeste de España y más específicamente en el suroeste de Galicia, en la frontera con el norte de Portugal. La muestra 
final fue de 236 empresas con más de 10 trabajadores y menos de 250 con un error muestral de $6,35 \%$.

Para definir las variables que intervienen en el modelo, se hizo uso de diferentes ítems (JARDON; MARTOS, 2010) medidos en una escala de Likert que iba desde 1 (no es importante como ventaja competitiva) hasta llegar al valor 5 (es muy importante como ventaja competitiva). Este tipo de valoración está basado en escalas contrastadas en la literatura (NARVER; SLATER, 1990; MALHOTRA, 1981, DESHPANDE; GOLHAR, 1994, CAMISÓN, 1999).

\section{RESULTADOS}

Para determinar la validez del análisis factorial se analiza la normalidad de los datos, mediante el test de Kolmogorov-Smirnov (NEWBOLD; CARLSON; THORNE, 2002). Si fallase la normalidad unidimensional también fallaría la multidimensional, puesto que la primera es condición necesaria de la segunda. Los resultados muestran que se rechaza la normalidad en todas las variables. Esto es lo que nos decide por el ACP. Utilizaremos indistintamente el nombre factor o componente una vez determinado el método.

\subsection{Elección del número de factores}

El siguiente paso consiste en decidir cuál es el número de factores con el que nos quedamos en el análisis. El método de Guttman-Kaiser selecciona 9 factores. La experiencia suele indicar que este número tiende a sobre estimar ligeramente el número de factores reales (COSTELLO; OSBORNE, 2005), por lo tanto será un límite superior.

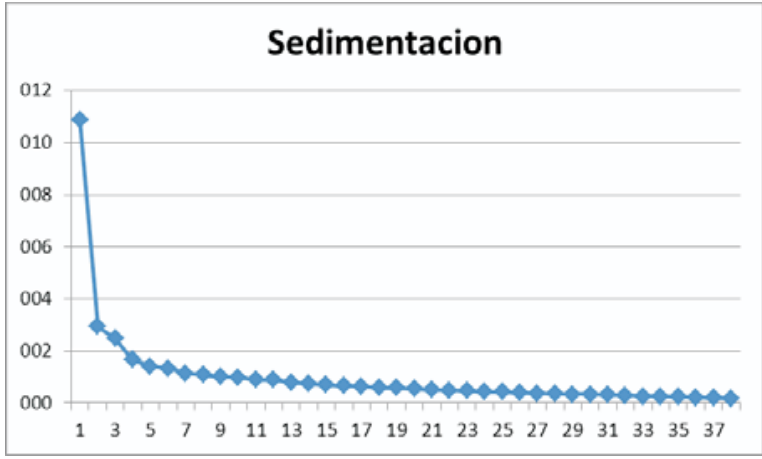

Figura 1

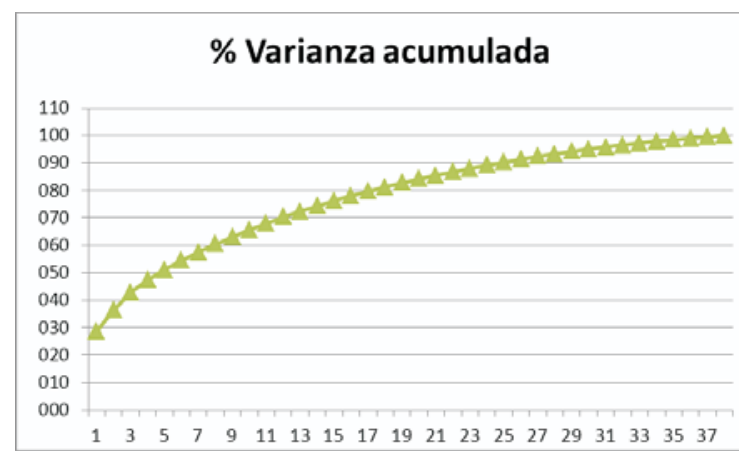

Figura 2

El segundo criterio considerado en los textos clásicos es el gráfico de sedimentación (KLINE, 2002) que se recoge en la figura 1. En él se observa que empieza suavizarse la caída a partir del tercero o cuarto factor. Utilizaremos esta cantidad como cota inferior del número de factores.

El otro elemento a tener en cuenta es el grafico de varianza explicada, puesto que nos indica la parte de información de las características de la empresa utilizada por ésta para generar las competencias distintivas (ver Figura 2). Se observa que para obtener un mínimo del $50 \%$ es necesario considerar al menos 5 factores. 
Con esta información hacemos un estudio de las comunalidades de las variables al variar el número de factores que introducimos en el modelo, para ver cuándo se puede considerar suficiente la información común a todos ellos. Se han calculado las comunalidades obtenidas desde 3 factores hasta 9 (ver Tabla 1 del anexo). Estas van aumentando ligeramente en algunos casos mientras que en otros dan pequeños saltos que indican la introducción de una nueva variable. Suponemos que una variable se introduce en el modelo si su comunalidad es mayor o igual que 0,4. Con 7 componentes introducimos todas las FVC pero ya con 5 estarían la mayoría. Esto nos sugeriría elegir entre 5 o 7 componentes.

Optamos inicialmente por elegir 5 competencias, aunque la interpretación y resultados finales pueden hacer variar algo esta conclusión previa. A pesar de que existen algunas variables que no llegan a tener la comunalidad mínima exigida, casi todas ellas están entre 0,35 y 0,4, salvo la calidad de los productos o servicios. Hemos probado a eliminarla y el modelo apenas variaba. Dado que tiene interés para explicar el conjunto, hemos decidido mantenerla.

\subsection{Selección del método de rotación}

En la rotación oblicua, se hicieron varias pruebas con diferentes valores de Delta. Los ensayos realizados con Deltas igual a 0 y negativos dieron como resultado que las iteraciones de la rotación no convergían. Nos quedamos finalmente con dos valores positivos.

La tabla 1 recoge la varianza explicada por cada componente después de la rotación. En las rotaciones ortogonales la varianza explicada total es la suma de las varianzas explicadas por cada componente, pero eso no ocurre en las rotaciones oblicuas. En estas últimas se mezclan los efectos de las diferentes variables al no tener correlaciones nulas. No obstante, de hecho en todos los casos, la varianza total explicada coincide con la que se obtiene antes de rotar los factores.

Una primera diferencia que se observa entre los diferentes métodos es el orden de las competencias. Esto no es un problema, puesto que solo indica que en unos casos explica menos que en otros la competencia obtenida, pero no cambia esencialmente su interpretación. Los métodos que consiguen competencias más equilibradas en cuanto a la varianza explicada son el método equamax entre los ortogonales y el método promax entre los oblicuos. Los que consiguen factores con mayor variación explicada, lógicamente ocurre cuanto más oblicuos son los factores, porque asumen varianza de unos y de otros. Eso ocurre con el método oblimin 0.5.

Tabla 1

\begin{tabular}{|c|c|c|c|c|c|c|}
\hline \multicolumn{7}{|c|}{ Varianza explicada } \\
\hline Factores & Varimax & Quartimax & Equamax & Oblimin 0.2 & Oblimi0.5 & Promax \\
\hline PC1 & 13.085 & 13.071 & 12.131 & 18.465 & 22.884 & 19.163 \\
\hline PC2 & 10.993 & 17.395 & 10.382 & 19.251 & 23.332 & 20.512 \\
\hline PC3 & 10.444 & 10.927 & 9.897 & 15.744 & 20.997 & 15.982 \\
\hline PC4 & 9.197 & 4.540 & 9.553 & 9.060 & 6.960 & 14.676 \\
\hline PC5 & 7.283 & 5.068 & 9.038 & 12.745 & 15.837 & 14.427 \\
\hline
\end{tabular}

La tabla 2 recoge el número de variables con cargas fuertes que interviene en cada factor. Se observa que en cada rotación oblicua aparecen dos matrices diferentes para interpretar los resultados. La mayoría de los métodos tienen el aproximadamente el mismo número de 
cargas factoriales fuertes en todos los factores, salvo el método Quartimax que presenta 11 en la segunda componente y 0 en la cuarta. Los otros dos métodos de rotación ortogonal presentan prácticamente las mismas variables y no se diferencia mucho su interpretación en uno u otro caso.

Las matrices de configuración se comportan de modo similar a las rotaciones ortogonales, mientras que la matriz de estructura muestra muchas más cargas factoriales, lo que indica que las competencias son formadas por una combinación más compleja de características. Observamos como el hecho de cambiar el Delta no afecta esencialmente a la rotación. En consecuencia sugerimos iniciar con un valor nulo o positivo y aumentarlo para ver los efectos que produce. Se debería elegir aquel que se adapte mejor a las hipótesis de partida, dando coherencia a la interpretación final.

Tabla 2

\begin{tabular}{|c|c|c|c|c|c|c|c|c|c|}
\hline Factores & Varimax & $\begin{array}{l}\text { Quartim } \\
\text { ax }\end{array}$ & $\begin{array}{l}\text { Equama } \\
\mathrm{x}\end{array}$ & $\begin{array}{l}\text { Oblimin } \\
0.2 \\
\text { Configura } \\
\text { ción }\end{array}$ & \begin{tabular}{|l} 
Oblimin \\
0.2 \\
Estructura \\
\end{tabular} & $\begin{array}{l}\text { Oblimin } \\
0.5 \\
\text { Configura } \\
\text { ción } \\
\end{array}$ & \begin{tabular}{|l} 
Oblimin \\
0.5 \\
Estructura \\
\end{tabular} & $\begin{array}{l}\text { Promax } \\
\text { Configur } \\
\text { ación } \\
\end{array}$ & $\begin{array}{l}\text { Promax } \\
\text { Estructura }\end{array}$ \\
\hline PC1 & 7 & 7 & 6 & 7 & 11 & 7 & 14 & 6 & 11 \\
\hline $\mathrm{PC} 2$ & 6 & 11 & 5 & 6 & 13 & 8 & 15 & 7 & 14 \\
\hline PC3 & 4 & 6 & 4 & 4 & 10 & 9 & 11 & 4 & 9 \\
\hline PC4 & 4 & 0 & 4 & 3 & 4 & 2 & 4 & 4 & 6 \\
\hline PC5 & 3 & 3 & 4 & 3 & 5 & 3 & 5 & 3 & 5 \\
\hline
\end{tabular}

En el anexo, se recogen las cargas factoriales tras las diferentes rotaciones. Se han seleccionado en todas las tablas aquellas celdas que tienen cargas factoriales fuertes. Se comprueba que la mayor parte de los métodos producen resultados similares, si bien los elementos que las componen difieren ligeramente. En algunos casos aparece con signo negativo y en otros, positivo pero eso no afecta a la interpretación. Esto nos confirma otra hipótesis de trabajo. El método de rotación no sesga marcadamente la interpretación del análisis de modo global. Sin embargo si se producen diferencias en algunos aspectos, sobre todo a la hora de indicar que características se asocian a cada componente. Dadas estas consideraciones consideramos apropiada la interpretación de las competencias obtenida en Jardon e Martos (2010).

\section{CONCLUSIONES}

Determinar las competencias distintivas de una empresa es fundamental para detectar las ventajas competitivas de ésta a la luz de la teoría de recursos y capacidades. (YOLLES, 2009). Esto es especialmente necesario para las pymes. En Latinoamérica son muy abundantes este tipo de empresas (DÍAZ; LORENZO; SOLÍS, 2005) por lo que es más necesario encontrar procedimientos de análisis. Aunque existen numerosos trabajos que detallan las competencias distintivas en la empresa, no se presenta una metodología común a todos ellos y eso hace difícil la comparación entre unos y otros. (CARSON; GILMORE, 2000; RUBIO; ARAGÓN, 2008). Una simplificación habitual es hacer uso de métodos multivariantes lineales. Dentro de esas técnicas se presentan dos aproximaciones: el análisis confirmatorio y el análisis exploratorio. Las competencias distintivas obtenidas mediante métodos exploratorios van a ser más realistas y 
más fácilmente adaptables a la situación específica de las empresas, puesto que permiten más flexibilidad, al recoger la asociación de los diferentes factores combinados en cantidades diversas en cada competencia.

Una vez analizados los diferentes métodos factoriales exploratorios se comprueba que apenas existen diferencias entre los análisis de componente principales y otros métodos factoriales en la práctica. Sin embargo, dado que este método recoge más información muestral y exige menos suposiciones inferenciales parece más conveniente para un análisis previo. (RAO, 1955).

También se analizan los diferentes procedimientos para seleccionar el número de competencias. En este caso se debe tener en cuenta el método de Guttman-Kaiser para determinar el número máximo, el grafico de sedimentación para determinar el número mínimo y otros métodos como la varianza aportada por cada factor, la varianza explicada total y la información que las competencias asumen de las diferentes características de la empresa para tomar una decisión inicial sobre dicho número. No obstante esta cantidad podrá revisarse a la vista de los factores obtenidos finalmente. (COSTELLO; OSBORNE, 2005).

Para evaluar si una característica puede ser considerada FVC se hace uso de la comunalidad de esa variable que nos indica la parte de la FVC que es utilizada para elaborar las competencias distintivas. Cuando una característica tiene una comunalidad muy baja (menos de 0 ,4) quiere decir que apenas se utiliza para elaborar competencias distintivas y por consiguiente puede ser eliminada de las FVC, coincidiendo con la mayoría de autores. (COSTELLO; OSBORNE, 2005). Este será uno de los criterios para indicar cuando una variable se puede considerar FVC, pero no es el único, puesto que hay que considerar su posible interpretación en el modelo final.

La rotación busca facilitar la interpretación de los factores obtenidos previamente. Para ello se intenta obtener una estructura factorial lo más simple posible. Dado que las competencias suelen estar relacionadas entre sí en la práctica, y que no existen grandes diferencias entre los diferentes métodos de rotación parece conveniente hacer uso de rotaciones oblicuas para determinar competencias. (STEWART, 1981).

Dentro de las rotaciones oblicuas, para interpretar el significado de cada competencia parece lógico hacer uso de la matriz de configuración para darle un primer nombre a la competencia pues suele tener una estructura más simple, pero para justificar las hipótesis de relaciones se debería utilizar la matriz de estructura puesto que es la que da información de las correlaciones entre características y competencias.

\section{REFERÊNCIAS BIBLIOGRÁFICAS}

ANDERSON, T. W. Asymptotic Theory for Principal Component Analysis. The Annals of Mathematical Statistics, v. 34, n. 1, p. 122-148, 1963.

ARTEAGA, M.; LASIO, V. Empresas dinámicas en Ecuador: factores de éxito y competencias de sus fundadores. Academia, Revista Latinoamericana de Administración, 42, p. 1-19, 2009.

BAI, J. Inferential Theory for Factor Models of Large Dimensions. Econometrica, v. 71, n. 1, p. 135-171, 2003.
BARNEY, J. The Resource-Based Theory of the Firm. Organization Science, v. 7, n. 5, p. 469-487, 1996.

BERMAN, S.; DOWN, J.; HILL, C. Tacit Knowledge as a Source of Competitive Advantage in the National Basketball Association. The Academy of Management Journal, v. 45, n. 1, p. 13-31, 2002.

BONTIS, N.; KNOW, W. C.; RICHARDSON, S. Intellectual capital and business performance in Malaysian industries. Journal of Intellectual Capital, v. 1, n. 1, p. 85-100, 2000. 
BROWN, J. Choosing the Right Type of Rotation in PCA and EFA. Shiken: JALT Testing y Evaluation SIG Newsletter, v. 13, n. 3, p. 20-25, 2009.

CAMISÓN, C. Sobre cómo medir las competencias distintivas: un examen empírico de la fiabilidad y validez de los modelos multi-item para la medición de los activos intangibles. The Iberoamerican Academy of Management. First international Conference. 9-11 de Diciembre. Madrid: Universidad Carlos III, 1999.

CARMELI, A. Strategic human capital and the performance of public sector organizations. Scandinavian Journal of Management, v. 20, n. 4, p. 375-392, 2004.

CARSON, D.; GILMORE, A. SME Marketing Management Competencies. International Business Review, v. 9, n. 3, 363-382, 2000.

CONFORTINI, D. ; LOPEZ, L. Qualitative assessment of stock prices listed on the São Paulo stock exchange: An approach from the perspective of homogeneity analysis. Academia Revista Latinoamericana de Administración, v. 42, p. 2033, 2009.

COSTELlO, A.; OSBORNE, J. Best Practices in Exploratory Factor Analysis: Four Recommendations for Getting the Most From Your Analysis. Practical Assessment, Research y Evaluation, v. 10, n. 7, p. 1-9, 2005.

CRONBACH, L. J. Coefficient alpha and the internal structure of tests. Psychometrika, 16(3), p. 297-334, 1951.

CHIN, W. The partial Least squares approach to structural equation model. In: M. G. A. Modern Methods for business Research. Mahwah, New Jersey, London: Lawrence Erlbaum, 1998.

DESHPANDE, S.; GOLHAR, D. HRM Practice in Large and Small Manufacturing Firms: A Comparative Study. Journal of Small Business Management, v. 32, n. 2, p. 49-56, 1994.

DÍAZ, A.; PÉREZ, C. Estrategias y prácticas logísticas y de tecnología de información: el caso de Venezuela. Academia. Revista Latinoamericana de Administración, v. 28, p. 5-26, 2002.
DÍAZ, A.; LORENZO, O.; SOLÍS, L. Procesos de negocios de Pymes insertas en redes colaborativas. Academia. Revista Latinoamericana de Administración, v. 34, p. 25-46, 2005.

GATTERMANN, M. et al. The moderating effects of innovativeness on new product development ability. Produto y Produção, v. 11, n. 3, p. 19-28, 2010.

GILMORE, A.; CARSON, D. Entrepreneurial Marketing By Networking. New England Journal Of Entrepreneurship, 12(2), p. 31-8, 1999.

GORSUCH, R. L. Factor analysis. Hillsdale: Lawrence Erlbaum, 1983.

GRANT, R. The Resource-Based Theory of Competitive Advantage: Implications for Strategy Formulation. California Management Review, v. 33, n. 3, p. 114-135, 1991.

GRANT, R. Contemporary Strategy Analysis. 5. ed. Oxford: Blackwell, 2005.

GUTTMAN, L. Some necessary conditions for common factor analysis. Psychometrika, v. 19, n. 2, p. 149-161, 1954.

HAFEEZ, K.; ZHANG, Y.; MALAK, N. Core competence for sustainable competitive advantage: a structured methodology for identifying core competence. IEEE Transactions on Engineering Management, v. 49, n. 1, p. 28-35, 2002.

HAIR, J. F. et al. Multivariate data analysis. 6. ed. Upper Saddle River: Pearson; Prentice Hall, 2006.

HARMAN, H. H. Modern Factor Analysis. Chicago: The University of Chicago Press, 1976.

HUGHES, P.; MORGAN, R. Fitting strategic resources with product-market strategy: Performance implications. Journal of Business Research, v. 4, n. 61, p. 323-331, 2008.

JARDON, C. M. et al. La cadena de la madera en el Departamento de Oberá (Misiones). Posadas: Universidad Nacional de Misiones (EDUNAM), 2007.

JARDON, C.; MARTOS, M. Competencias basicas en Pymes regionales: El caso de Vigo y su área de influencia. Revista galega de Economia, 2010. (En prensa) 
KAISER, H. An index of factorial simplicity. Psychometrika, v. 39, n. 1, p. 31-36, 1974.

KAISER, H. F. Image analysis. In: HARRIS, C. W. Problems in measuring change. Madison: University of Wisconsin Press, 1963. p. 156-166.

KETELHÖHN, W.; MONCAYO, M.; ALLEN, B.¿Realmente existe la competitividad nacional? Academia. Revista Latinoamericana de Administración, v. 22, p. 59-67, 1999.

KLINE, P. An easy guide to factor analysis. London: Routledge, 2002.

LAWLEY, D. N.; MAXWELL, A. E. Factor Analysis as a Statistical Method. Journal of the Royal Statistical Society, Series D (The Statistician), v. 12, n. 3, p. 209-229, 1962.

MALAVER, F.; VARGAS, M. Los procesos de innovación en América Latina: aportes para su caracterización / Hacia una caracterización de los procesos de innovación en la industria colombiana. Los resultados de un estudio de casos. Academia Revista Latinoamericana de Administración, v. 33, p. 5-33, 2004.

MALHOTRA, N. A scale to measure self concepts and product concepts. Journal of Marketing Research, v. 18, n. 4, p. 456-464, 1981.

NARVER, J.; SLATER, S. The Effect of a Market Orientation on Business Profitability. Journal of Marketing, v. 54, n. 4, p. 20-35, 1990.

NEWBOLD, P.; CARLSON, W.; THORNE, B. 5. ed. Statistics for Business and Economics. New York: Prentice Hall, 2002.

O'DONNELL, A. et al. Competitive Advantage In Small To Medium Sized Enterprises. Journal Of Strategic Marketing, v. 10, n. 3, p. 205-223, 2002.

PÉREZ, M. P.; PRIETO, I. M. ; MARTÍN. Gestionando el conocimiento a través de la gestión de recursos humanos: análisis empírico en el sector de automoción. Academia. Revista Latinoamericana de Administración, v. 42, p. 49-71, 2009.

PETT, M.; SULLIVAN, J.; LACKEY, M. Making Sense of Factor Analysis: the Use of Factor Analysis for Instrument Development in Health Care Research. London: SAGE, 2003.
PRAHALAD, C.; HAMEL, G. The Core Competence of the Corporation. Harvard Business Review, v. 687, n. 3, p. 79-91, 1991.

RAO, C. Estimation and tests of significance in factor analysis. Psychometrika, v. 20, n. 2, p. 93-111, 1955.

RAY, G.; BARNEY, J.; MUHANNA, W. Capabilities, Business Processes, And Competitive Advantage: Choosing The Dependent Variable In Empirical Tests Of The Resource-Based View. Strategic Management Journal, v. 25, n. 1, p. 23-37, 2004

RUBIO, A.; ARAGÓN, A. Recursos estratégicos en las pymes. Revista Europea de Dirección y Economía de la Empresa, v. 17, n. 1, p. 103-126, 2008.

STEWART, D. The Application and Misapplication of Factor Analysis in Marketing Research. Journal of Marketing Research, v. 18, n. 1, p. 51-62, 1981.

TABACHNICK, B. G.; FIDELL, L. S. Using Multivariate Statistics. Boston: Allyn and Bacon, 2001.

THURSTONE, L. L. Multiple factor analysis: a development and expansion of vectors of the mind. Chicago: University of Chicago Press, 1947.

TINSLEY, H.; TINSLEY, D. Uses of Factor Analysis in Counseling Psychology Research. Journal of Counseling Psychology, v. 34, n. 4, p. 414-424, 1987.

VAONA, A.; PIANTA, M. Firm size and innovation in European manufacturing. Small Business Economics, v. 30, n. 3, p. 283-299, 2008.

VELICER, W. F.; FAVA, J. L. Effects of variable and subject sampling on factor pattern recovery. Psychological Methods, v. 3, n. 2, p. 231-251, 1998.

VELICER, W. F.; JACKSON, D. N. Component Analysis Versus Common Factor-Analysis - Some Further Observations. Multivariate Behavioral Research, v. 25, n. 1, p. 97-114, 1990.

YOLLES, M. Competitive advantage and its conceptual development: an exploration. Business Information Review, v. 26, n. 2, p. 93-111, 2009. 


\section{Anexos}

Tabla 3 - Listado de características

\begin{tabular}{|c|c|}
\hline$\ldots$ & Nombre de la característica \\
\hline V001 & 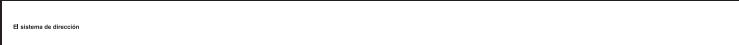 \\
\hline V002 & La formación de los directivos y trabajadores \\
\hline V003 & La profesionalidad y actitud de los trabajadores \\
\hline V004 & La cultura de la empresa \\
\hline V005 & La actitud de cooperación y alianzas de la empresa \\
\hline V006 & La capacidad de innovación del personal \\
\hline V007 & La capacidad de evaluación de riesgos \\
\hline V008 & Las tecnologías de procesos y productos utilizadas \\
\hline V009 & Los recursos humanos \\
\hline V010 & Los recursos financieros \\
\hline V011 & Los recursos tecnológicos de equipos \\
\hline V012 & Los recursos naturales \\
\hline V013 & El conocimiento del mercado \\
\hline V014 & El tipo de clientes \\
\hline V015 & La atención al cliente \\
\hline V016 & El sistema de fidelización de clientes \\
\hline V017 & El tiempo de respuesta a las necesidades del cliente \\
\hline V018 & La relación directa con los clientes finales \\
\hline V019 & El servicio posventa \\
\hline V020 & La red de distribución \\
\hline V021 & La promoción de los productos/servicios \\
\hline V022 & El precio que tienen los productos/servicios \\
\hline V023 & La amplitud de la cartera de productos/servicios \\
\hline V024 & La exclusividad de los productos/servicios \\
\hline V025 & La calidad de los productos/servicios \\
\hline V026 & El proceso de investigación/desarrollo/innovación \\
\hline V027 & El proceso de diseño \\
\hline V028 & El sistema de información \\
\hline V029 & La comunicación interna \\
\hline V030 & El tipo de proveedores \\
\hline V031 & El proceso de aprovisionamiento \\
\hline V032 & El sistema de producción \\
\hline V033 & El proceso de comercialización y venta \\
\hline V034 & El sistema de calidad \\
\hline V035 & Las áreas geográficas en las que compra \\
\hline V036 & Las áreas geográficas en las que produce \\
\hline V037 & Las áreas geográficas en las que vende \\
\hline V038 & Capacidad de penetración en nuevos mercados \\
\hline
\end{tabular}


Tabla 4 - Comunalidades para diferente número de competencias

\begin{tabular}{|c|c|c|c|c|c|c|c|}
\hline Comunalidades & PCA3 & PCA4 & PCA5 & PCA6 & PCA7 & PCA8 & PCA9 \\
\hline V001 & ,481 & 510 & ,555 &, 560 & ,647 & ,647 & ,650 \\
\hline V002 &, 578 & ,605 & ,610 &, 610 & ,648 & ,650 & 651 \\
\hline V003 &, 619 & ,642 & ,642 & ,645 & ,669 & ,673 &, 673 \\
\hline V004 &, 520 &, 522 & ,536 &, 541 &, 549 &, 550 &, 551 \\
\hline V005 &, 320 & ,354 & ,390 & ,416 & ,418 & ,486 & ,711 \\
\hline V006 & ,461 &, 530 & ,597 & ,624 & ,624 & ,624 & ,668 \\
\hline V007 &, 371 &, 413 & ,483 &, 591 & ,597 & ,608 & ,624 \\
\hline V008 & ,441 &, 548 &, 550 &, 632 & ,633 & ,634 &, 690 \\
\hline V009 &, 537 &, 540 & ,571 &, 574 &, 590 &, 599 &, 599 \\
\hline V010 & ,287 & ,287 & ,560 &, 582 &, 586 &, 589 &, 645 \\
\hline V011 &, 416 & ,467 & ,488 &, 581 & ,591 & ,601 & ,682 \\
\hline V012 &, 351 & ,351 & ,357 & ,387 &, 460 &, 499 &, 545 \\
\hline V013 &, 421 & ,426 & ,426 &, 434 &, 434 &, 458 & ,485 \\
\hline V014 &, 360 &, 366 & ,366 &, 384 &, 488 & ,604 & 614 \\
\hline V015 & ,664 &, 673 & ,678 &, 714 & ,721 &, 723 &, 726 \\
\hline V016 &, 552 & ,632 & ,656 & ,689 & ,695 & ,702 & ,704 \\
\hline V017 & ,382 & ,383 & ,383 & ,434 &, 520 & ,524 &, 550 \\
\hline V018 & ,399 & ,451 & ,451 &, 502 &, 506 &, 508 &, 509 \\
\hline V019 & ,357 & ,474 & ,483 & ,498 &, 531 & ,538 & ,561 \\
\hline V020 &, 515 &, 531 & ,531 & ,542 & ,656 & 660 & ,674 \\
\hline V021 & ,507 &, 510 & ,560 & ,602 & ,651 & ,651 & ,657 \\
\hline V022 &, 312 &, 506 & ,523 & 600 & ,600 & ,619 & ,624 \\
\hline V023 & ,385 & ,478 & ,496 & ,643 & ,644 & ,684 & ,685 \\
\hline V024 & ,380 & ,399 & ,475 &, 494 &, 506 &, 514 &, 531 \\
\hline V025 & ,290 & ,312 & ,317 &, 319 & ,400 & ,577 &, 591 \\
\hline V026 & ,381 & ,572 & ,727 & ,741 & ,755 & ,755 & ,760 \\
\hline V027 & ,318 & ,495 & ,647 & ,666 & ,668 & ,685 & ,686 \\
\hline V028 & ,449 & ,495 & ,525 & ,525 &, 528 & ,532 &, 540 \\
\hline V029 & ,486 &, 552 & ,555 &, 569 &, 579 & ,580 &, 589 \\
\hline V030 & ,404 & ,448 & ,459 & ,496 & ,501 & ,599 &, 599 \\
\hline V031 & ,516 & ,540 & ,545 & ,625 & ,650 & ,701 &, 733 \\
\hline V032 & ,383 & ,445 & ,469 &, 591 &, 591 & ,619 & ,648 \\
\hline V033 & ,496 & ,498 & ,508 &, 512 &, 599 & ,617 & ,617 \\
\hline V034 & ,339 & ,379 & 381 & ,383 &, 527 & ,563 & ,612 \\
\hline V035 & ,458 & ,460 & ,510 &, 576 &, 580 & ,606 & ,633 \\
\hline V036 & ,466 & ,466 & ,536 &, 565 & ,608 & 684 & ,686 \\
\hline V037 & ,280 & ,284 & ,394 & ,406 & ,443 & 600 & ,702 \\
\hline V038 & ,418 &, 426 & ,439 & ,463 & ,464 & ,481 &, 543 \\
\hline
\end{tabular}


Tabla 5 - Cargas factoriales en las rotaciones

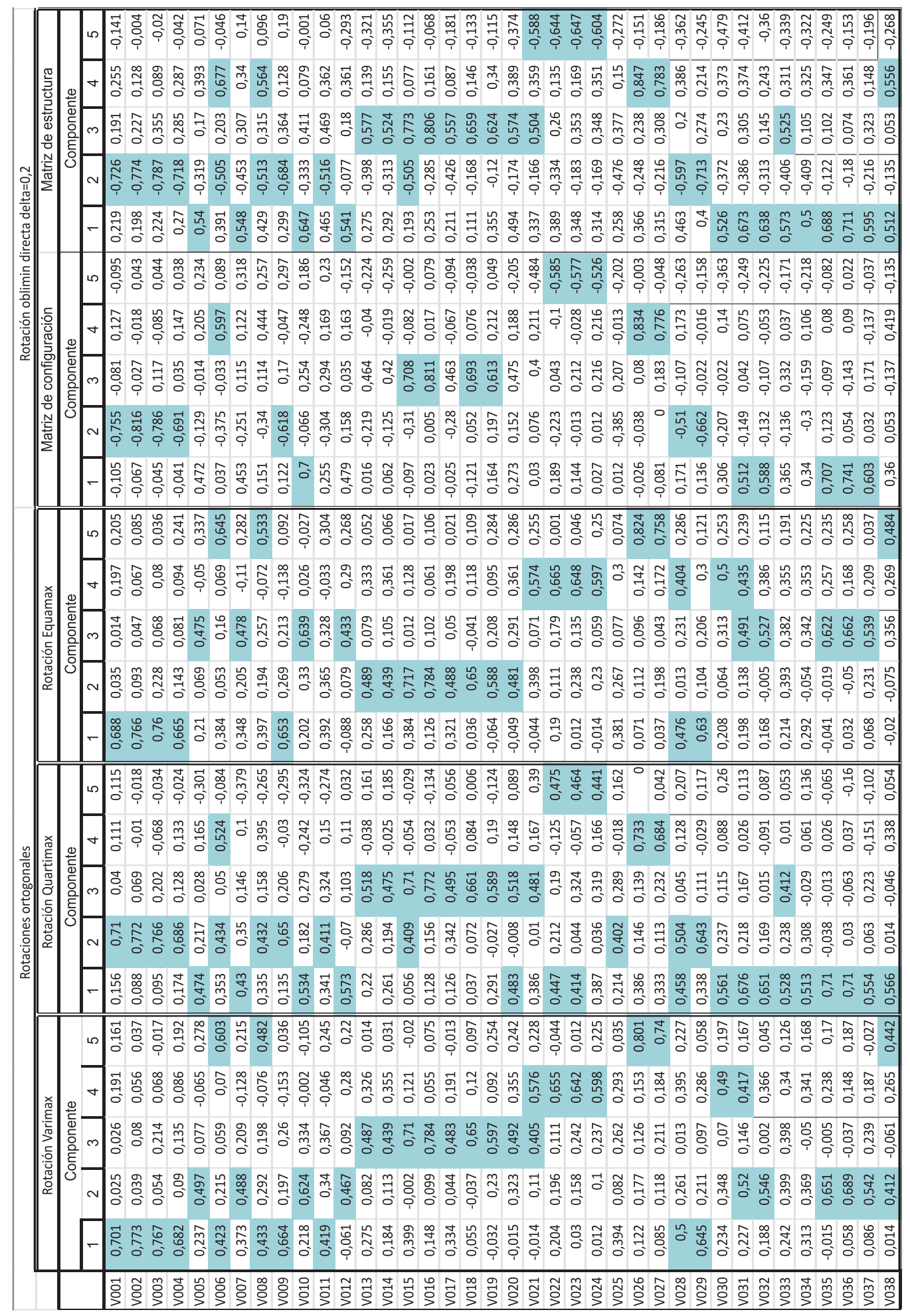


Tabla 5 - Cargas factoriales en las rotaciones (continuación)

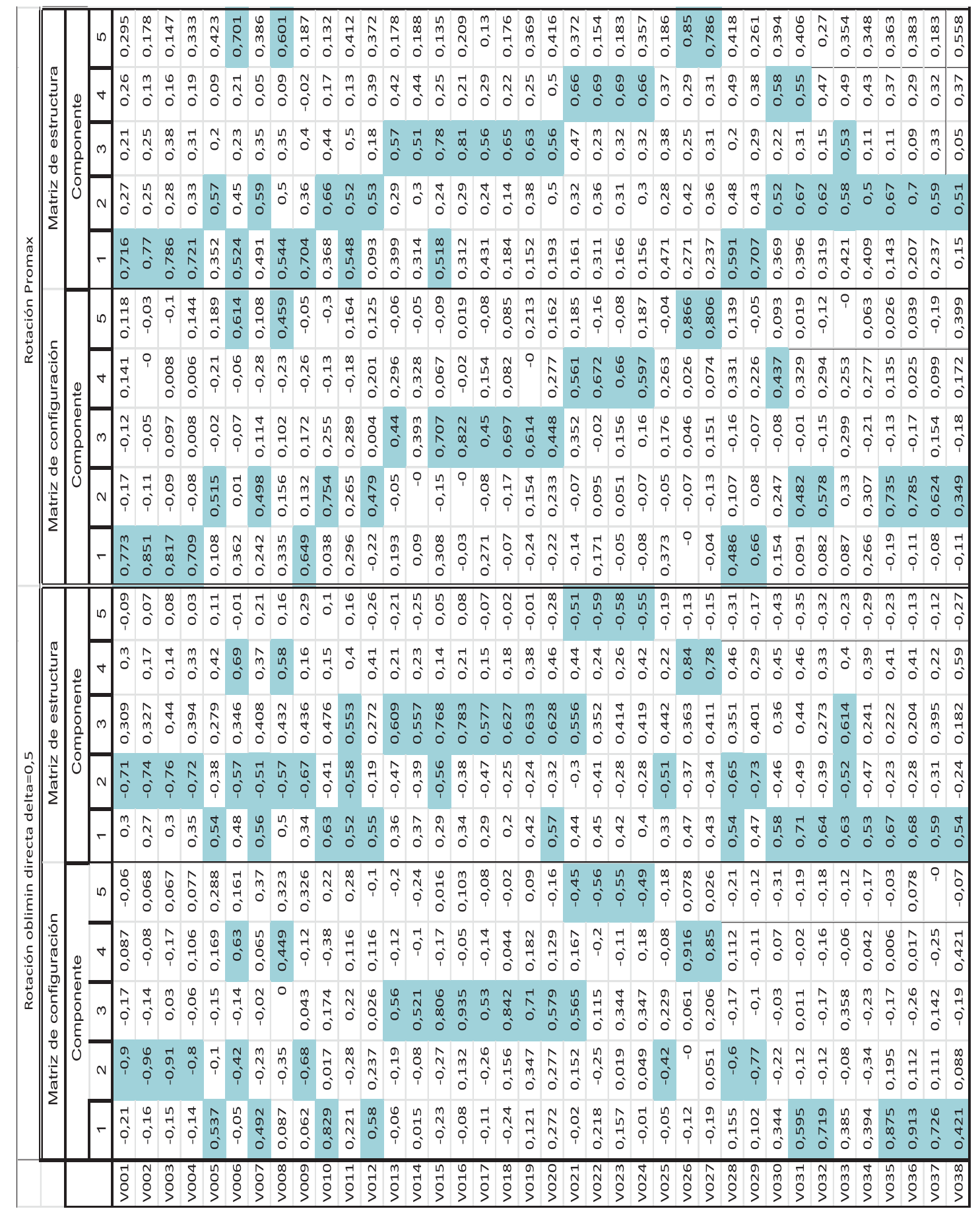

